\title{
Assessing the impact of Physicians' Virtual Communities on their medical Decision Making quality
}

\author{
Anjum Razzaque \\ Ahlia University, College of Business and Finance, \\ Manama, Bahrain \\ anjum.razzaque@gmail.com
}

\author{
Tillal Eldabi \\ Brunel University, Brunel Business School, College \\ of Business, Arts and Social Sciences \\ tillal.eldabi@brunel.ac.uk
}

\begin{abstract}
Medical decision making is daunting to physicians of its unclear benefits for improving patient care while such decisions are evidence based and also are from the social capital of resources of the advises shared between their peers. Past scholars have reported great deal of medical errors and misdiagnoses caused by physicians: a situation that is degrading healthcare quality. It is not surprising why past research also stressed on the importance to empirically explore the effect of physicians' virtual community on their medical decision making quality. Virtual communities are a promising initiative in the healthcare sector. This paper describes how the participation of VC members is possible through the application of the Social Capital Theory's three dimensions in order to assess the effectiveness of physicians' virtual community so they can make better quality of medical decisions. Such is depicted in this paper's conceptual model. The model was empirically tested for its validity and reliability using an adapted survey for which data was collected from 204 SurveyMonkey virtual community physician members. The empirical evidence supports the hypothesis of the conceptual model through physicians' identification and shared vision, i.e. two pre-requisites for medical DM.
\end{abstract}

\section{Introduction:}

Physicians' made medical errors have increased healthcare (HC) costs and raised patient mortality rates [15]. Such errors occurr during quick evidence-basedmedical decision making (DM). While such DM is through assembling and interpreting of information, physicians' DM is insufficient, even though such DM is based on evidenced-reasoning. This is since, DM is made during hectic situations [34]. This is why physicians have begun to desire making medical decisions through inquiry and support by sharing experiences in virtual communities (VCs) [18, 40, 41].
On one hand, from VC's perspective, a VC environment is a self-supported cyberspace network where participants communicate in forums or chat rooms [28, 31]. Physicians can harness the power of $\mathrm{VCs}$ for joint patient-care DM since VCs are KM strategies [55]. This is a social networking concept regaining research attention. VC Interactions are motivated through discussions with mutual goals and interests. This way, VCs build social capital (SC) of resources, which in turn explain VC members' participation. In this case, the SC of resources are ideas, emotional support, etc. [1, 7, 21, 25, 26, 32, 41]. On the other hand, from the perspective of medical $\mathrm{DM}, \mathrm{DM}$ is an important $\mathrm{HC}$ research topic since clinicians' frequently make diagnostic errors and such a circumstance has highlighted clinical reasoning an under researched area; even though this research area has existed since 60 years [13]. Clinical reasoning is an important factor for consideration since it provides evidence-based-accuracy during medical DM [27].

Hence, this study aims to assess the effect of physicians' VCs, through physicians' SC of resources in VCs, on their DM quality; since research lacks to analyze the effect of knowledge management (KM) tools on HC topics [45] where DM is the HC topic of this study. DM is in need of further research considering that diagnostic errors degrade medical DM quality. DM requires multiple inputs from various stakeholders for accurate DM through experiences shared in a VC, where VCs are facilitating KM tools [5, 12, 22, 32, 16, 48]. Also, such an assessment fulfills the need to integrate the technological and the social perspective prescribed for future research [19]. This is, along with, the need for more quantitative research desired to assess the effectiveness of VCs; such that, this study also performed quantitative analysis to assess its research aim. This is in line with what [18] reports that very few studies have assessed the effectiveness of a community of practice.

Next, Section 2 provides a theoretical background of the literature reviewed to relate SCT and DM. Subsequently, followed by the 6 sub-hypotheses critiqued and proposed in Section 3; Section 4, 
justifies the methodology this study implemented to test its hypotheses. This is followed by the description of the data analyses and discussions of the empirical results, along with their implications revealed in Section 6 and 7.

\section{Theoretical Background:}

SCT wise; past research expressed significant interest on the role of SC in VCs [19] while applying SCT in topics like anthropology, economics, management and political science [1]. Current research assessed users' behaviors using SCT to explain social participation, in order to appreciate the benefits of VC members' bonding of relations to attain human and financial SC of reserves; for competitive advantage [19, 21, 25]. This is similar to how customer comments are the SC of resources for an organization, when its customers partake in a VC to offer feedback $[7,8,40]$. Recent research aggregated SCT with other theories like the Technology Acceptance Model, to judge why VC members voluntarily participate [19]. Such research also applied SCT to express trust, relations and communications between VC members during their voluntary participation [40]. SC is reflective in a $\mathrm{VC}$ when its members benefit from their personal and business relations, governed by their norms and cultures [1]. SCT's three dimensions: structural, relational and cognitive dimension were applied in this study. The structural dimension expresses overall relationships through the social interaction ties (SIT). The relational dimension is the nature of relations expressed through trust, norms of reciprocity (NoR) and identification (ID). The cognitive dimension is the common understanding through shared vision (SV) and shared language (SL) [7].

Medical DM wise; DM theory existed since 1960s. Research in medical DM primarily focuses on the role of physicians' DM [13] where accurate DM means accountable evidence-based-practice. Research has expressed DM through terminologies like clinical DM, diagnostic reasoning, clinical judgment, clinical inference and problem solving. Clinical DM is situational, since the choice of a decision is out of alternative decisional outcomes. During clinical DM, information is processed in a situational, rational and logical evidence-based-practice. The practitioner articulates supporting decisional knowledge for DM $[27,58]$. Ample DM occurs during the diagnosing process where poor diagnoses cause poor recommendations [33]. Hence, DM is a set of sequential activities: clear problem identification, solutions classification, alternative solutions analysis, appropriate action planning and adapted solution assessment [50]. In this case, DM is a choice-basedtreatment where DM can be a: (1) professional choice (decided by clinician based on patient's consent), shared DM (both clinician and patient decide) or consumer choice (patient decides based on clinician shared information) [16]. Since two decades, research explained the importance of technology for DM, like DM occurring in social networks. Traditional DM is based on an uncertain yet possible actions based on experience and reasoning [3]. Treatment DM is associated with clinical DM where a clinical decision is guided by evidence: evidence-based-DM; were evidence and personal experience harmoniously work together $(44,51]$. Even though diagnostic DM is critical, it is a seldom addressed area [13].

There is a clear rationale why this study aims to assess the relation between SCT and DM. As [26] reported, scholars addressed research questions from three perspectives: the social perspective (members' collective deeds to participate in a VC), the technical perspective (use of technology to express the $\mathrm{VC}$ environment) and the socio-technical perspective (merging of the technical and social perspectives). The issue is that on one hand several studies assessed the social perspective to investigate the importance of VCs while ignoring the technical perspective; while on the other hand there are the other studies that assessed VC's technical perspective while ignoring the social perspective. Under such circumstances, this study can apply the SCT perspective to extend the aim of recent research, by applying the socio-technical perspectives of VCs to assess the effect of physicians' SC on their medical DM quality. This is such that, the social perspective of VCs is the application of the SCT and the $\mathrm{VC}$ environment of physicians, being a $\mathrm{KM}$ tool, is the technical perspective in this study.

\section{Research Hypotheses and Model:}

$\mathrm{SC}$ of resources are decision aids vital for DM and basis for organizational learning since DM outcomes are experiences added to SC of resources, during SITs. I.e. positive / negative DM outcome adds to further experience towards the SC of resources $[17 ; 36]$. SCT's three dimensions are composed of six factors: SI), Trust, NoR, ID, SL and SV [7; 8]. Hence, the study's main hypothesis $\mathbf{H 1}$ is: Physicians' SC positively and significantly affects DM in a VC. This hypothesis was developed by theoretical but not empirical support since this is the first study empirically assessing H1. Prior research only went so far as to assess the role of SCT on shared knowledge [7. 8]. $\mathrm{H} 1$ is based on 6 sub-hypotheses ( $\mathrm{H}$ 1a to $\mathrm{H}$ 1f) depicted in Figure 1. 
SIT wise; VC members' interactions log evidences of shared experiences aiding DM. Complex problem solving and DM occur during interactions [58]. Doctors prefer DM through interactions with VC members to share experiences to create SC of resources during $\mathrm{DM}$, i.e. during bonding when interacting with VC member [19, 21, 25, 36, 44, 45]. Hence, sub-hypothesis H 1a is: Physicians' SIT positively and significantly affect DM in a VC. Trust wise; trust stirs confidence during DM since one can trust group's verdict even though such DM is risky and complex. This is acceptable since DM is based on choices where clinicians can manage risks [24, 37, 44, 47, 51]. Trust aids mutually benefiting managers, during their social ties, to boost DM; making trust a pre-requisite for DM [2, 42]. Trust reduces members' uncertain actions and improves their innovation and confidence [44]. Hence, sub-hypothesis $\mathbf{H} \mathbf{1 b}$ is: Physicians' Trust positively and significantly affects $\mathrm{DM}$ in a $\mathrm{VC}$.

NoR wise; effective DM involves effective information processing through interconnected participants, within network's norms and standards where group norms are standards effective during group DM: especially during problem solving [42, 49]. Hence sub-hypothesis H 1c is: Physicians' NoR positively and significantly affects DM in a VC. ID wise; VC members' ID aids communication for mutual purpose through discussions during shared DM. Employees identify themselves with organizational values and objectives, similarly with a
VC and its members. Consequently, DM is aided by ID. Organizations prefer employees who strongly identify with their goals. Employees also prefer identifying with their organizations. Employees, with higher identification with organizations indulge in topmanagement aligned DM. ID is a sense of belonging in a $\mathrm{VC}$, which endures participation [7, 8, 20, 23]. Hence, sub-hypothesis H 1d is: Physicians' ID positively and significantly affects DM in a VC.

SL wise; SL promotes problem-solving during DM. [44] assessed how managers use knowledge for ICT related DM and reported that participative thinking and analyzing are central for understanding and expressing a problem. Communication, using SL, is critical for DM during a learning process. Language manages conflicts in cross-cultural teams and supports strategic DM. SL is a largely ignored research topic $[39,46]$. Hence, sub-hypothesis H 1e is: Physicians' SL positively and significantly affects DM in a VC. SV wise; DM stresses on the need for collaboration essential for HC related networks $[14,51]$ where HC admission related DM requires $\mathrm{SV}$ of staff members so clinicians can manage risks. DM is based on relevant choices, which, in turn are based on meaning and values. In the HC sector, admission related DM is complex were difficult DM is based on insufficient patient information; since more than $75 \%$ of participants wish they had made different decisions. When working with staff, teamwork requires SV for reach a collective view to aid DM $[11,24]$. Hence, the sub-hypothesis H 1f is: Physicians' SV positively and significantly affects DM in a VC.

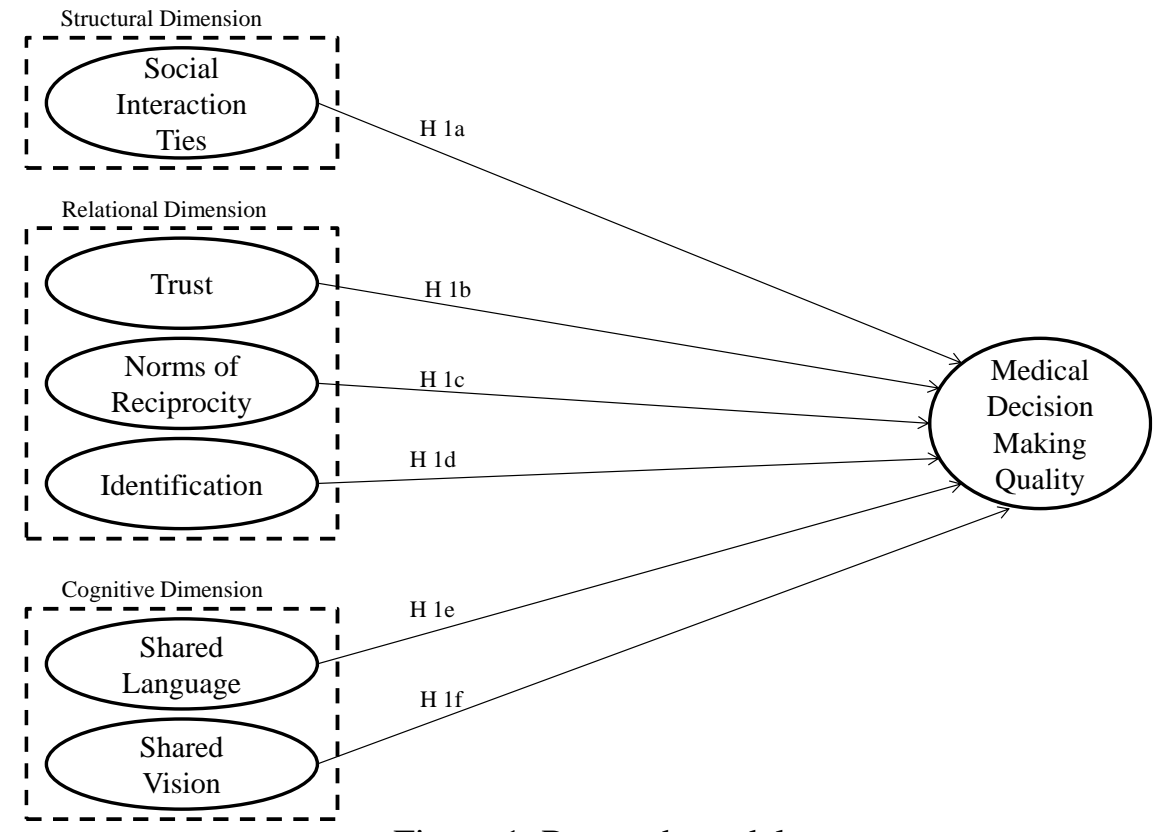

Figure 1. Research model 


\section{Research Methodology:}

This study initiated with a thorough literature review (LR), which resulted in: the scholars publishing a number of articles [56] and identifying a research aim: to assess the relationship between VC physicians' SCT and DM (Figure 1's research model depicting six sub-hypotheses expressing the research aim). The model was further tested using a questionnaire, which was assessed for its reliability and validity using a 5-point-Likert-scale (Stronglyagree to Strongly-disagree). Figure 1 depicts the DM as the dependent variable (DV), composed of 6 items adapted from [58], also listed in Table 2. The six SCT independent variables (IVs), SIT, trust, NoR, ID, SL and SV are composed of 22 items adapted from [7, 8], also listed in Table 2. All IVs are individually itemize SCT and expressed in three dimensions (structural, relational and cognitive): a demonstration also adapted from $[7,8]$. Figure 1 clearly depicts the 6 hypotheses sub-(H 1a - H 1f).

Furthermore, while the instrument reflected SCT's 22 items and DM's 6 items, its first part articulated the purpose and the nature of this research project and expressed an agreement to maintain confidentiality of its data. First, the questionnaire received 31 responses when pilot tested in the "plastic_surgery@yahoogroups.com" VC of plastic surgeons'. The pilot study results led the instrument to be amended for grammatical mistakes while all items were left as is, since they were reported strongly reliable. Since various dimensions of the instrument were adapted from various studies $[7,8,58]$, the questionnaire was not tested for its validity during the pilot study phase.

Next, the survey [52] was hosted on SurveyMonkey (SM), and was distributed to 600 SM's VC physician member from the US [29] and consequently received 204 responses $(n=204)$. After the commencement of the data collection phase, data analysis was performed using Confirmatory Factor Analysis (CFA), followed by Structural Equation Modeling (SEM). The results of the data analysis are discussed in the next section.

\section{Results}

The first part of the survey articulated the research purpose, its nature and a memorandum of understanding to maintain confidentiality of its data. After the 31 responses from the pilot study, the instrument received 204, of 600, complete responses.
Henceforth, the frequency \% and respondents' count, from the first part of the survey, is depicted in Table 1. Instrument reliability and validity was performed through two steps. The first step involved the analyses of the conceptual model using CFA and the second step involved the testing of the structural relationships of this study's conceptual model using SEM.

CFA assessed the instrument reliability of its seven scales (SIT, T, NoR, ID, SL, SV and DM) using LISREL. Every item of the CFA model reflected its latent constructs, where all seven constructs co-varied. Maximum likelihood approach was utilized to estimate the model with item-to-item-correlation as an input. CFA results are depicted in Table 2. In order, for the conceptual model, to achieve model fitness, various indices were calculated and thus depicted, with recommended acceptable values, in Table 3. In addition, the scale's convergent validity was confirmed by Factor Loading, Construct Reliability and Average Variance Extracted (as depicted in Table 2). CFA wise: all Factor Loading, Construct Reliability and Average Variance Extracted (AVE) values surpassed acceptable value range; making the scale pass convergent validity.

The next step was to assess the scale's discriminant validity, i.e. assessing the construct's square root of AVE with satisfactory value recommended to surpass the correlation of that construct in relation with other constructs of this study's model [8]. Table 4 depicts the correlation values amid constructs and the square root of AVE in a diagonal format with an observation that these AVEs surpass construct correlation values between constructs: hence assuring instrument construct validity. SEM wise: the structural model tested linear relationships between constructs. The model was deemed fit as the acceptable indices values surpassed acceptable threshold (as depicted in Table $3)$.

Hypotheses results (depicted in Figure 2) concluded that 1 of 6 paths demonstrating $\mathrm{P}$ value < 0.01 while 1 of 6 paths displayed $P$ value $<0.001$. The remaining paths were insignificant, i.e. at a 0.05 significance level. Hence, SV and ID exhibited a strong positive and significant effect on DM quality. SIT, T and NoR proved insignificant with DM quality. Hence, hypothesis $\mathrm{H} 1 \mathrm{~d}$ and $\mathrm{H} 1 \mathrm{f}$ were supported while hypothesis $\mathrm{H} \mathrm{1a}, \mathrm{H} \mathrm{1b}, \mathrm{H} 1 \mathrm{c}$ and $\mathrm{H}$ 1e were not supported by this study's empirical results. 
Table 1. Demographics $(n=204: \mathrm{n}$ refers to response rate)

\begin{tabular}{|c|c|c|c|}
\hline \multirow[t]{2}{*}{ Measure } & \multirow[t]{2}{*}{ Items } & \multicolumn{2}{|c|}{ Frequency } \\
\hline & & Response \% & Respondent's count \\
\hline \multirow[t]{2}{*}{ Gender } & Male & $72.1 \%$ & 147 \\
\hline & Female & $27.9 \%$ & 57 \\
\hline \multirow{5}{*}{$\begin{array}{l}\text { Work } \\
\text { experience (in } \\
\text { years) }\end{array}$} & Less than 5 & $20.1 \%$ & 41 \\
\hline & $5-10$ & $9.3 \%$ & 19 \\
\hline & $11-15$ & $7.4 \%$ & 15 \\
\hline & $16-20$ & $13.7 \%$ & 28 \\
\hline & Above 20 & $49.5 \%$ & 101 \\
\hline \multirow{15}{*}{$\begin{array}{l}\text { Specialty (i.e. } \\
\text { department) }\end{array}$} & Internal Medicine & $12.5 \%$ & 24 \\
\hline & General Surgery & $3.6 \%$ & 7 \\
\hline & OBS/GYN & $5.2 \%$ & 10 \\
\hline & Pediatrics & $8.9 \%$ & 17 \\
\hline & Family Medicine & $12.5 \%$ & 24 \\
\hline & Ophthalmology & $1.6 \%$ & 3 \\
\hline & Dermatology & $1 \%$ & 2 \\
\hline & ENT & $0.5 \%$ & 1 \\
\hline & Radiology & $0.5 \%$ & 1 \\
\hline & Anesthesiology & $3.1 \%$ & 6 \\
\hline & Physiotherapy & $1 \%$ & 2 \\
\hline & Urology & $0.5 \%$ & 1 \\
\hline & Neurology & $1 \%$ & 2 \\
\hline & Emergency & $4.7 \%$ & 9 \\
\hline & Other (please specify) & $43.2 \%$ & 83 \\
\hline \multirow{6}{*}{$\begin{array}{l}\text { I am part of a } \\
\text { VC because I } \\
\text { am part of } \\
\text { a/an: }\end{array}$} & Professional Email list & $59.5 \%$ & 103 \\
\hline & Professional group in a social media platform, e.g. & $24.5 \%$ & 50 \\
\hline & Facebook, LinkedIn or Twitter & & \\
\hline & Professional platform on the Internet e.g. SERMO, & $22.5 \%$ & 46 \\
\hline & QuantiaMD, Epocrates, etc & $2.5 \%$ & 5 \\
\hline & $\begin{array}{l}\text { Video conference for joint discussion or collaboration } \\
\text { between two or more physicians }\end{array}$ & & \\
\hline
\end{tabular}

Table 2. Summary of measurement scale \& Reliability Analysis $(n=204)$ to Assess Convergent Validity

\begin{tabular}{|c|c|c|c|c|c|c|c|}
\hline $\begin{array}{c}\text { Measured } \\
\text { items }\end{array}$ & $\begin{array}{c}\text { Item-to-total } \\
\text { correlation }\end{array}$ & $\begin{array}{c}\text { Factor } \\
\text { loading }\end{array}$ & $\begin{array}{c}\text { Composite } \\
\text { reliability }(\mathrm{CR})\end{array}$ & AVE & Cronbach's ó & Mean & $\begin{array}{l}\text { Std. } \\
\text { Dev. }\end{array}$ \\
\hline SIT & & & 0.86 & 0.75 & 0.851 & & \\
\hline SI_1 & 0.745 & 0.91 & & & & 2.59 & 1.149 \\
\hline SI_2 & 0.745 & 0.82 & & & & 2.36 & 1.021 \\
\hline Trust & & & 0.79 & 0.56 & 0.782 & & \\
\hline $\mathrm{T} \_1$ & 0.532 & 0.58 & & & & 3.07 & 0.857 \\
\hline T_3 & 0.721 & 0.80 & & & & 3.11 & 0.784 \\
\hline $\mathrm{T} \_4$ & 0.621 & 0.84 & & & & 3.29 & 0.825 \\
\hline NoR & & & 0.92 & 0.86 & 0.921 & & \\
\hline N_1 & 0.855 & 0.95 & & & & 3.67 & 0.683 \\
\hline N_2 & 0.855 & 0.90 & & & & 3.72 & 0.655 \\
\hline ID & & & 0.94 & 0.84 & 0.936 & & \\
\hline I_1 & 0.879 & 0.93 & & & & 3.04 & 0.925 \\
\hline I_2 & 0.816 & 0.94 & & & & 2.88 & 0.884 \\
\hline I_3 & 0.827 & 0.86 & & & & 3.09 & 0.879 \\
\hline SL & & & 0.78 & 0.64 & 0.766 & & \\
\hline SL_1 & 0.627 & 0.71 & & & & 3.83 & 0.637 \\
\hline SL_2 & 0.627 & 0.88 & & & & 3.81 & 0.554 \\
\hline SV & & & 0.83 & 0.62 & 0.831 & & \\
\hline SV_1 & 0.671 & 0.75 & & & & 3.69 & 0.637 \\
\hline SV_2 & 0.703 & 0.83 & & & & 3.64 & 0.711 \\
\hline SV_3 & 0.701 & 0.78 & & & & 3.54 & 0.714 \\
\hline DM & & & 0.90 & 0.75 & 0.892 & & \\
\hline DMQ_1 & 0.791 & 0.86 & & & & 3.18 & 0.657 \\
\hline DMQ_3 & 0.848 & 0.94 & & & & 3.22 & 0.685 \\
\hline DMQ_5 & 0.730 & 0.78 & & & & 3.20 & 0.707 \\
\hline
\end{tabular}


* Item-to-total correlation assesses instrument validity: minimal acceptable value of 0.5 [48].

* Factor loading: minimum acceptable value should be $>0.5$ [7]

* Composite reliability assesses construct reliability [8] and convergent validity [35]: minimal acceptable value should be 0.7 [35].

* Cronbach's ó: assess construct's constructs' reliability [9]: minimum acceptable value should be greater than 0.6 [7].

* AVE: assess internal consistency [10] and convergent validity [35]: minimal acceptable value $\geq 0.5$ [7, 35].

* Minimum reliability (CR) should exceed 0.7 [7].

* Items in the survey:

- $\quad$ SI_1: I maintain close social relationships with some members in a VC.

- $\quad$ SI_2: I spend a lot of time interacting with some members in the VC on a personal level.

- SI_3: I know some members in a VC on a personal level.

- SI_4: I have frequent communication with some members in the VC.

- I_1: Members in a VC will not take advantage of others even when the opportunity arises.

- I_2: Members in a VC will always keep the promise they make to one another.

- I_3: Members in a VC would not knowingly do anything to disrupt the conversation.

- I_4: Members in a VC behave in a consistent manner.

- I_5: Members in a VC are truthful in dealing with one another.

- $\quad$ N_1: I know that other members in a VC will help me, so it's only fair to help other members.

- $\quad$ N_2: I believe that members in the VC would help me if I need it.

- I_1: I feel a sense of belonging towards the VC.

- I_2: I have the feeling of togetherness or closeness in the VC.

- I_3: I have a strong positive feeling towards the VC.

- I_4: I am proud to be a member of the VC.

- SL_1: Members in the VC use common terms or jargons.

- SL_2: Members in the VC use understandable communication pattern during the discussion.

- SL_3: Member in the VC use understandable narrative forms of post messages or articles.

- $\quad$ SV_1: Members in the VC share the vision of helping others solve their professional problems.

- $\quad$ SV_2: Members in the VC share the same goal of learning from each other.

- $\quad$ SV_3: Member in the VC share the same value that helping others is pleasant.

- DMQ_1: I am very certain of the diagnoses after my interaction with members in virtual community.

- DMQ_2: I am very certain of the treatment after my interaction with members in virtual community.

- DMQ_3: I am very certain of the health benefits after my interaction with members in virtual community.

- DMQ_4: I am very certain of the side effects after my interaction with members in virtual community.

- DMQ_5: I am very certain of the risks after my interaction with members in virtual community.

- DMQ_6: I am very certain of the use of evidence-based knowledge after my interaction with members in virtual community.

Table 3: Model Fitness

\begin{tabular}{lcl}
\hline \multicolumn{1}{c}{ Model fit indices } & Results of this study & Recommended values \\
\hline Chi square $\left(\mathrm{x}^{2}\right)$ "normalized by degrees of freedom $(\mathrm{CMIN}=$ & 1.77 & $\leq 3[7]$ \\
201/48/DF $=114)$ & & \\
CFI - Comparative Fit Index & 0.98 & $\geq 0.9[8]$ \\
NNFI - Non-Normed Fit Index & 0.98 & $\geq 0.9[38]$ \\
RMSEA - Root Mean Square Error of Approximation & 0.061 & $\leq 0.08$, \\
& & i.e. sensible good fit [54] \\
\hline
\end{tabular}

\begin{tabular}{cccccccccc} 
& \multicolumn{8}{c}{ Table 4: } & \multicolumn{8}{c}{ Descriptive Statistics and Correlation from Constructs } & & \\
& Mean & S.D. & SIT & T & NoR & ID & SL & SV & DM \\
SIT & 2.474 & 2.028 & $\mathbf{0 . 8 7}$ & & & & & & \\
T & 3.154 & 2.060 & 0.41 & $\mathbf{0 . 7 5}$ & & & & & \\
NoR & 0.694 & 1.289 & 0.45 & 0.74 & $\mathbf{0 . 6 5}$ & & & & \\
ID & 3.002 & 2.532 & 0.67 & 0.58 & 0.56 & $\mathbf{0 . 9 2}$ & & & \\
SL & 3.822 & 1.075 & 0.31 & 0.56 & 0.64 & 0.44 & $\mathbf{0 . 8}$ & & \\
SV & 3.623 & 1.784 & 0.38 & 0.68 & 0.73 & 0.57 & 0.68 & $\mathbf{0 . 7 9}$ & \\
DM & 3.201 & 1.860 & 0.44 & 0.39 & 0.50 & 0.59 & 0.46 & 0.56 & $\mathbf{0 . 8 7}$
\end{tabular}

* SD - Standard Deviation.

* Diagonal element (in bold) are square root of the variance extracted (VE). Off-=diagonal elements are correlations between constructs. To assess discriminant validity the diagonal elements should be $>$ off-diagonal elements.

* Correlation Coefficients were assessed via CFA model. All are significant, i.e. $p<0.05$ as observed in [7]. 


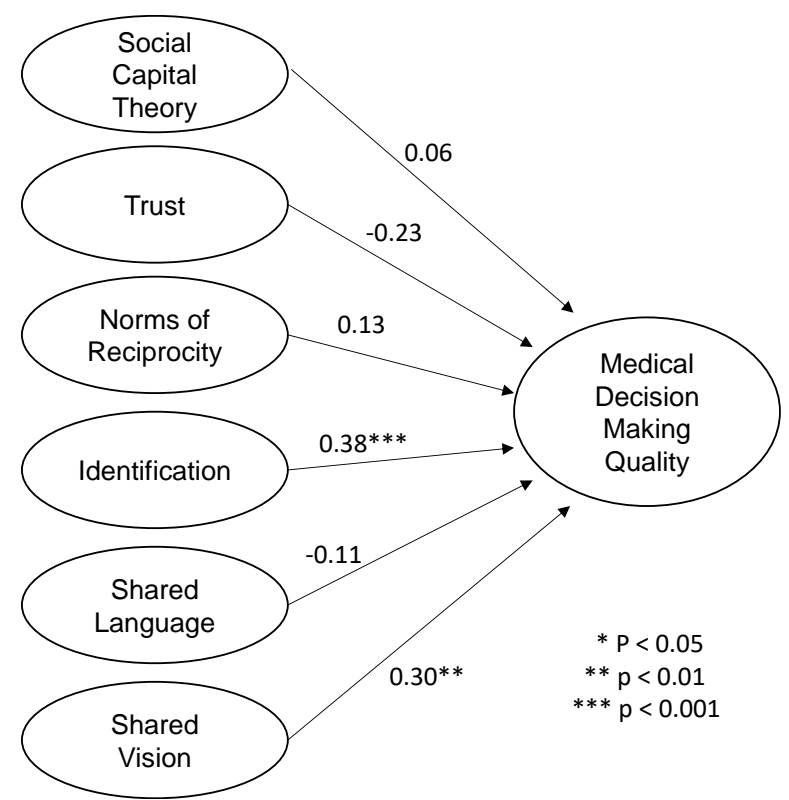

Figure 2. SEM Analysis results from LISREL.

\section{Discussion and Limitations:}

SEM analysis of Figure 2's empirical evidence confirmed SCT influences DM through VC members' ID and SV since ID and SV positively and significantly affected DM quality. Hence, SCT'S ID and $\mathrm{SV}$ are pre-requisites for DM quality. In contrast, SCT's Trust and SL played a negative role on DM while SCT's SIT and NoR expressed no significance on DM. Even though Trust is a prerequisite of DM in a VC, Figure 2's empirical findings indicate that a VC does not motivate trust for DM, since trust negatively facilitated DM. One explanation is that the interpersonal aspect of electronic networks make trusting a challenging task [37].

Studies advocating a significant role of ID and SV on DM $[4,6,11,20,23,24]$ were supported by this study. From this study's empirical evidence (Figure 2 SEM analysis) SIT, NoR and SL played an insignificant role on VC DM. Even though past research advocated a positive and a significant relationship between SIT and DM [36, 44, 51] this study's empirical evidence confirmed otherwise. Also, it is not surprising that NoR played an insignificant role on medical DM since even though trust and NoR affiliate with one another, ample theory warns that exchange seldom offers positive outcomes, i.e. shared DM [4]. Also, scholars advocating that SL facilitates DM [44] were not supported by this study's model. Since physicians experience language barriers in varying cultures, like during advice-giving; this is why
SL was confirmed an insignificant role on DM. Such a problem hampers shared DM between physicians and patients. Still, with regards to the role of SL on $\mathrm{DM}$; more research is required to assess why SL is insignificant during VC DM. Also, it is not surprising why [53] reported that research lacks to explore how language and culture barriers affect shared DM. In conclusion; this study's empirical evidence suggests that the only reason why SCT facilitates medical DM is due to VC members' ID and SV and not because of their interaction, norms, trust or common language.

Even though this study expressed a promising and valuable empirical evidence, it has limitations. Despite its empirical evidence assessing its literature-driven model on a SM VC of physician members; it is still unclear if this empirical evidence can apply over other professional VCs. Another research limitation is that this study could have been influence by self-selection bias, since this study's participants' sample size was based on VC member of active VC participants but not those who may have ended their participation. Those may differ in opinion on VC's SC.

Although data was collected for the IVs and DV of this study's models at the same time using the same instrument; such an cross-sectional data collection prompts Common Method Bias (CMB), To confirm if the study's instrument suffered from CMB, Harman's one-factor test applied on this study's data using SPSS. Considering that Harman's one-factor test revealed only one factor ensuring FA accounting at $43.662 \%$ variance, even though high, this is an acceptable value since it is less than 50\% for acceptable CMB [57]. Future research should conduct more advance CMB tests using CFA and to collect IV and DV related data at different times; to avoid/reduce CMB further.

The study's empirical evidence is based on SM VC physicians. Additional research can assess the root cause of the generalization of these empirical results. Data collection was cross-sectional causing this study to miss out to investigate time-rich enduring phenomenon to enhance its empirical findings. Further investigation could cater a longitudinal time-rich study for HC VCs. Another possible limitation, suggested by [30], is that more study should be led outside U.S. to authenticate published findings of the US.

\section{Research and Practice Implications:}

This study's empirical findings provide a deeper understanding of the influence of various facets of SCT on medical DM, in a VC. From the theoretical 
perspective, the empirical findings furthered the understanding that not all SCT factors significantly affect DM, in a VC: when particularly affiliating with physicians VCs. In addition to the assessment of SCT on DM, this study narrowed the research gap, as [45] recommended to analyze the effectiveness of $\mathrm{KM}$ tools on HC topics. Future research could assess these relations longitudinally, as also suggested by [8]. Finally, while some studies, e.g. [7, 8], assessed the relation between SCT and knowledge sharing; this study narrowed a gap reported by another study, i.e. recommending future research to assess the impact of a VC in the absence of a knowledge sharing behavior [50]. The authors of this study successfully assessed the influence of a VC in the absence of the knowledge sharing behavior, since this study shed light on the empirical results expressing the influence of various facets of SCT on DM (Figure 2).

Till now, medical DM research has focused on emergency treatment, chronic disorders treatment and palliative care. Here chronic diseases research, e.g. cancer, showed interest in medical DM. Globally, DM research contributed in areas like assessing physicians' role in complex settings and patients' roles as well as roles of care givers' who are actively involved in the treatment [50]. Alternatively, clinical practices involve thinking and DM. Even though diagnostic DM is critical, it is a seldom-addressed topic. Now that diagnostic errors frequently occur with uncertain diagnoses, thinking and DM got further research attention [13].

Hence, it is time that the findings of this study be considered for practical implementation by the $\mathrm{HC}$ sector, such that the empirical wisdom from this study could be utilized for improving strategic HC organizational objectives, protocols and strategies, which in turn could encourage physicians to indulge in $\mathrm{DM}$ based on policies and protocols that harness the $\mathrm{VC}$ environment in parallel to protocols of evidence based DM, This way, there would be a practical understanding of [43]'s reported disadvantage of SCT, i.e. to understand why some VC members benefit more than others. One possible reason could be the variations in the organizations where their social structures dominate members' choices for seeking resources within their ties. Yet, this rational also needs practical and empirical assessment.

\section{References}

[1] Akdere, M. "Social Capital Theory and Implications for Human Resource Development", Singapore Management Review, 27(2), 2005, pp. 1-24.
[2] Alge, B. J., Wiethoff, C., \& Kleinc, H. J. "When does the medium matter? Knowledge-building experiences and opportunities in decision-making team", Organizational Behavior and Human Decision Processes, 91(1), 2003, pp. 26-37.

[3] Anya, O., Tawfik, H., Nagar, A., \& Amin, S. "ContextAware Decision Support in Knowledge-Intensive Collaborative E-Work", Procedia Computer Science, 1, 2010, pp. 2281-2290.

[4] Austin, J. R. "Transactive Memory in Organizational Groups: The Effects of Content, Consensus, Specialization, and Accuracy on Group Performance", Journal of Applied Psychology, 88(5), 2003, pp. 866-878.

[5] Bate, S., \& Robert, G. "Knowledge Management and Communities of Practice in the Private: Lessons for Modernizing the National Health Service England and Wales", Public Administration, 80(4), 2002, pp. 643-663.

[6] Bhattacharya, C. B., \&Sen, S. "Consumer-Company Identification: A Framework for Understanding Consumers' Relationships with Companies", Journal of Marketing, 67(2), 2003, pp. 76-88.

[7] Chang, H. H., \& Chuang, S.-S. "Social capital and individual motivations on knowledge sharing: Participant involvement as a moderator", Information \& Management, 48(1), 2011, pp. 9-18.

[8] Chiu, C.-M., Hsu, M.-H., \& Wang, E. T. "Understanding knowledge sharing in virtual communities: An integration of social capital and social cognitive theories", Decision Support Systems, 42(3), 2006, pp. 1872-1888.

[9] Chow, W. S., \& Chan, L. S. "Social network, social trust and shared goals in organizational knowledge sharing”, Information \& Management, 45(7), 2008, pp. 458-465.

[10] Churchill, G. A. "A Paradigm for Developing Better Measures of Marketing Constructs", Journal of Marketing Research, 16(1), 1979, pp. 64-73.

[11] Collins-Camargo, C., \& Hall, J. "How Creating a Shared Vision Can Improve More Than Outcomes”, Policy \& Practice, 68(3), 2010, pp. 10.

[12] Cook, D. A. "Medical Decision Making: What Do We Trust?", Journal of General Internal Medicine, 25(4), 2010, pp. 282-283.

[13] Croskerry, P., \& Nimmo, G. "Better clinical decision making and reducing diagnostic error", Journal of the Royal College of Physicians of Edinburgh, 41(2), 2011, pp. 155-162.

[14] D'Amour, D., Goulet, L., Labadie, J.-F., MartínRodriguez, L. S., \&Pineault, R. "A model and typology of collaboration between professionals in healthcare 
organizations", BMC Health Services Research, 8(1), 2008, pp. $188-202$.

[15] DeMarco, B. "I am wondering if people understand what they are paying for healthcare now, and what they are receiving in return?" Sept 2013, http://www.alzheimersreadingroom.com/2010/03/cost-ofhealthcare-worldwide.html

[16] Demiris, G. "The diffusion of virtual communities in health care: Concepts and challenges", Patient Education and Counseling, 62(2), 2006, pp. 178-188.

[17] Dovey, K., \& White, R. "Learning about learning in knowledge-intense organizations", The Learning Organization, 22(3), 2005, pp. 246-260.

[18] Eysenbach, G., Powell, J., Englesakis, M., \& Rizo, C. "Health related virtual communities and electronic support groups: systematic review of the effects of online peer to peer interactions", July 26, 2013, http://bmj.com/cgi/pmidlookup?view=long\&pmid=151429 21.

[19] Faraj, S., Jarvenpaa, S. L., \& Majchrzak, A. "Knowledge Collaboration in Online Communities", Organization Science, 22(5), 2011, pp. 1-16.

[20] Fiol, C. M., \& O'Connor, E. J. "Identification in Faceto-Face, Hybrid, and Pure Virtual Teams: Untangling the Contradictions", Organization Science, 16(1), 2005, pp. 1932.

[21] Florin, J., Lubatkin, M., \& Schulze, W. "A Social Capital Model of High-Growth Ventures', Academy of Management Journal, 46(3), 2003, pp. 374-384.

[22] Foong, D. P., \& McGrouther, D. A. "An Internetbased discussion forum as a useful resource for the discussion of clinical cases and an educational tool", Indian Journal of Plastic Surgery, 43(2), 2010, pp. 195-197.

[23] Gossett, L. M. "Kept at Arm's Length: "Questioning the Organizational Desirability of Member Identification", Communication Monographs, 69(4), 2002, pp. 385-404.

[24] Grounds, A., Gelsthorpe, L., Howes, M., Melzer, D., Tom, B. D., Brugha, T., et al. "Access to medium secure psychiatric care in England and Wales. 2: A qualitative study of admission decision-making", The Journal of Forensic Psychiatry \& Psychology, 15(1), 2004, pp. 32-49.

[25] Guillen, L., Coromina, L., \& Saris, W. E. "Measurement of Social Participation and its Place in Social Capital Theory", Social Indicators Research, 100, 2011, pp. 331-35.

[26] Ham, J., Park, J., Lee, J.-N., \& Moon, J. Y. "Understanding Continuous Use of Virtual Communities: A Comparison of Four Theoretical Perspectives" 45th Hawaii International Conference on System Sciences, 2012, pp. 753-762.
[27] Hancock, H. C., \& Durham, L. "Critical care outreach The need for effective decision-making in clinical practice (Part 1)", Intensive and Critical Care Nursing, 23(1), 2007, pp. 15-22.

[28] Hara, N., Solomon, P., Kim, S.-L., \& Sonnenwald, D. H. "An Emerging View of Scientific Collaboration: Scientists' Perspectives on Collaboration and Factors that Impact Collaboration", Journal of the American Society for Information Science and Technology, 54(10), 2003, pp. 952-965.

[29] Helft, M. "How Dave Goldberg turned Online Questtionnaires into a Hot Business", Fortune, 164(5), 2011, pp. 68-79.

[30] Herndon, N. C., Fraedrich, J. P., \& Yeh, Q. L. “An investigation of moral values and the ethical content of the corporate culture: Taiwanese versus U.S. sales people", Journal of Business Ethics, 30(1), 2001, pp. 73-85.

[31] Huq, A. "Engaging Organisational Culture to Overcome Social Barriers in Virtual Communities. In Encyclopedia of virtual communities and technologies, Washington DC: George Washington University, 2006, pp. 193-197

[32] Huysman, M., \& Wulf, V. "IT to support Knowledge sharing in communities, towards a social capital analysis", Journal of Information Technology, 21(1), 2006, pp. 40-51.

[33] Ismael, K. "Mekerene University Research Repository Item 123456789/628”, July 24, 2011, http://hdl.handle.net/123456789/628

[34] Jalal-Karim, A., \& Balachandran, W." Interoperability Standards: the most requested element for the Electronic Hea15lthcare Records significance", 2nd IEEE International Conference - E-Medical Systems, Tunisia: 2008.

[35] Jeon, S., Kim, Y.-G., \& Koh, J. "An integrative model for knowledge sharing in communities-of-practice", Journal of Knowledge Management, 5(2), 2011, pp. 251-269.

[36] Jansen, R. J., Curseu, P. L., Vermeulen, P. A., Geurts, J. L., \& Gibcus, P. "Social capital as a decision aid in strategic decision-making in service organizations", Management Decision, 49(5), 2011, pp. 734-747.

[37] Jøsang, A. “Trust-based decision making for electronic transactions", The 4th Nordic Workshop on Secure IT Systems, Stockholm University Report, 1999, pp. 99-005.

[38] Judge, T. A., Bono, J. E., \& Locke, E. A. "Personality and Job Satisfaction: The Mediating Role of Job Characteristics", Journal of Applied Psychology, 85(2), 2000, pp. 237-249. 
[39] Lauring, J., \& Selmer, J. "Multicultural organizations: common language, knowledge sharing and performance", Personnel Review, 14(3), 2011, pp. 324 - 343.

[40] Lin, K.-Y., \& Lu, H.-P. "Intention to Continue Using Facebook Fan Pages from the Perspective of Social Capital Theory", Cyberpsychology, Behavior and Social Networking, 14(10), 2011, pp. 565-570.

[41] Liu, C, Jiang, Z, Zhen, L. \& Su, H. "A bilateral integrative health-care knowledge service mechanism based on MedGrid", Medline, 38(4), 2008, pp. 446-460.

[42] Magnier-Watanabe, R., Yoshida, M., \& Watanabe, T. "Social network productivity in the use of SNS", Journal of Knowledge Management, 14(6), 2010, pp. 910-927.

[43] Marcum, C. S. "Unanticipated Gains: The Origins of Network Inequality in Everyday Life", Conttemporary Sociology, 39(3), 2010, pp. 348-349.

[44] Mascia, D., \& Cicchetti, A. "Physician social capital and the reported adoption of evidence-based medicine: Exploring the role of structural holes", Social Science \& Medicine, 72(5), 2011, pp. 798-805.

[45] Nicolini, D., Powell, J., Conville, P., \& MartinezSolano, L. "Managing knowledge in the healthcare sector. A review", .International Journal of Management Reviews, 10(3), 2008, pp. 245-263.

[46] Oxoby, R. J., \& McLeish, K. N. "Sequential decision and strategy vector methods in ultimatum", Economics Letters, 84(3), 2004, pp. $399-405$.

[47] Parayitam, S. "The effect of competence-based trust between physicians and administrative executives in healthcare on decision outcomes", Management Research Review, 33(2), 2010, pp. 174-191.

[48] Paul, D. L. "Collaborative Activities in Virtual Settings: A Knowledge Management Perspective of Telemedicine", Journal of Management Information Systems, 22(4), 2006, pp. 143-176.

[49] Postmes, T., Spears, R., \& Cihangir, S. "Quality of Decision Making and Group Norms", Journal of Personality and Social Psychology, 80(6), 2001, pp. 918930.

[50] Puschner, B., Steffen, S., Slade, M., Kaliniecka, H., Maj, M., Fiorillo, A., Munk-Jørgensen, P., Larsen, J. I., Égerházi5, A., Nemes, Z., Rössler, W., Kawohl, W., \& Becker, T. "Clinical Decision Making and Outcome in Routine Care for People with Severe Mental Illness (CEDAR): Study protocol viewing options: Abstract", BMC Psychiatry, 10(1), 2010, pp. 90.

[51] Sifer-Rivière, L., Girre, V., Gisselbrecht, M., \& SaintJean, O. 'Physicians' perceptions of cancer care for elderly patients: A qualitative sociological study based on a pilot geriatric oncology program" Critical Reviews in
Oncology/Hematology, 75(1), 2010, pp. 58-69.

[52] "Welcome to SurveyMonkey", Aug 10, 2012, http://www.surveymonkey.net/home/

[53] Suurmond, J., \& Seeleman, C. "Shared decisionmaking in an intercultural context Barriers in the Interaction between physicians and immigrant patients", Patient Education and Counseling, 60(2), 2006, pp. 253259.

[54] Yang, B., Watkins, K. E., \& Marsick, V. J. "The Construct of the Learning", Human Resource Development Quarterly, 15(1), 2004, pp. 31-55.

[55] Razzaque, A., Jalal-Karim, A., \& Karolak, M. "Culture sustained Knowledge Management Infrastructure and Architecture Facilitate Medical Decision Making", 2nd IEEE International Conference - IIAS-IASIA Joint Congress, Bahrain: 2013.

[56] Razzaque, A. “Anjum Razzaque” Sept 2017, https://ahliauniversity.academia.edu/AnjumRazzaque/JOU RNAL-PAPERS [Accessed 2 Sept 2017]

[57] Fuller, C. M. et al., "Common Methods variance detected in business research", Journal of Business Research, 69(8), 2016, pp. 3192-3198.

[58] Lin, C., \& Chang, S. "A relational model of medical knowledge sharing and medical decision-making quality", .International Journal of Technology Management, 43(4), 2008, pp. 320-348. 